\title{
Percepção de dor no parto normal em gestantes
}

A gestação é um período em que o corpo feminino sofre variadas alterações, sendo elas físicas e psíquicas. O parto é um evento que desperta nos gestantes sentimentos de angústia, medo, ansiedade e dor. O presente artigo foi realizado através de um levantamento bibliográfico realizado por meio de uma revisão de literatura de caráter qualitativo, considerando a relevância do tema. Notou-se uma grande rejeição por parte das mulheres em optarem pelo parto normal, devido às dores sentidas no trabalho de parto e no parto. Porém, perceberam-se também pontos relevantes na optação pelo parto normal; ele traz benefícios para a mãe e para o bebê, pois minimiza os riscos para a saúde de ambos os envolvidos em gestações de baixo risco, além de o parto natural ser recomendado pela Organização Mundial de Saúde (OMS). Notou-se, com esta pesquisa, que a fisioterapia pode auxiliar para a qualidade do trabalho de parto, facilitando em todas as suas fases, com técnicas que podem ajudar aliviando a dor, permitindo que a parturiente tenha melhor consciência corporal, prevenindo lesões no assoalho pélvico, e auxiliando na recuperação no pós-parto. É papel do fisioterapeuta esclarecer às gestantes quanto aos tipos de parto e todas as possibilidades existentes. Percebeuse também que o fisioterapeuta pode ser especializado em saúde da mulher, trabalhando com gestantes, auxiliando e orientando para que a mesma estimule a potencialidade do seu corpo no trabalho de parto e parto prevenindo complicações. A abordagem fisioterapêutica terá resultados benéficos sobre o bem-estar da gestante, ajudando e prevenindo maiores lesões na musculatura do assoalho pélvico nos partos normais.

Palavras-chave: Parto natural; Gestação; Fisioterapia; Humanização.

\section{Perception of pain in normal delivery in pregnant women}

Gestation is a period in which the female body undergoes various changes, both physical and psychic. Childbirth is an event that awakens in pregnant women feelings of anguish, fear, anxiety and pain. The present article was carried out through a literature review carried out through a qualitative literature review, considering the relevance of the theme. There was a great rejection by the women in opting for the normal birth, due to the pains in labor and delivery. However, we also noticed relevant points in the choice of normal delivery; it brings benefits to the mother and the baby as it minimizes the health risks of both those involved in low-risk pregnancies and natural childbirth is recommended by the World Health Organization. It was noted with this research that physical therapy can help the quality of labor, facilitating in all its phases, with techniques that can help alleviate pain, allowing the woman to have a better body awareness, preventing injuries to the floor pelvic recovery, and assisting in postpartum recovery. It is the role of the physiotherapist to explain to pregnant women the types of childbirth and all the possibilities that exist. It was also realized that the physiotherapist can be specialized in the health of the woman, working with pregnant women, helping and directing her to stimulate the potentiality of her body in labor and delivery preventing complications. The physiotherapeutic approach will have beneficial results on the well-being of the pregnant woman, helping and preventing greater lesions in the pelvic floor musculature in normal deliveries.

Keywords: Natural childbirth; Gestation; Physiotherapy; Humanization.

Topic: Fisioterapia

Reviewed anonymously in the process of blind peer.

Amanda Cristielle Pereira da Mata Oliveira

Faculdade Guaraí, Brasil

http://lattes.cnpq.br/2724961956698642

amandacristielle520@hotmail.com

Laize Batista de Sene

Faculdade Guaraí, Brasil

http://lattes.cnpq.br/0354240636601860

laizeicaro152015@hotmail.com

Luiz Aurélio Rodrigues Watanabe

Faculdade Guaraí, Brasil

http://lattes.cnpq.br/6877886751156526

luizrwatanabe@gmail.com

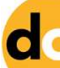

DOI: 10.6008/CBPC2236-9600.2018.002.0004
Received: 07/08/2019

Approved: 18/08/2019
Referencing this:

OLIVEIRA, A. C. P. M.; SENE, L. B.; WATANABE, L. A. R.. Percepção de dor no parto normal em gestantes. Scire Salutis, v.8, n.2, p.32-42, 2018. DOI: http://doi.org/10.6008/CBPC2236-9600.2018.002.0004 


\section{INTRODUÇÃO}

A gestação é um período em que o corpo feminino sofre variadas alterações, sendo elas físicas e psíquicas. Sendo o parto um evento que desperta nas gestantes sentimentos de angústia, medo, ansiedade e dor. As grávidas possuem opções de escolha da forma do parto, tendo a cesárea, que consiste em uma intervenção cirúrgica, tendo como indicação especifica somente em casos de riscos a gestante ou ao feto, e tem o parto vaginal que é um método natural de nascimento, baseado na saída do bebê pelo canal vaginal, não possuindo nenhuma intervenção cirúrgica e com uma rápida recuperação (LUIS et al., 2017).

O parto normal, até o início do século XX, era realizado por parteiras em domicilio, somente na década de 1940 que houve avanços tecnológicos e científicos, no qual se transformou em um evento intervencionista realizado em hospitais com a presença de profissionais da saúde (ABREU et al., 2013). Contudo, o parto vaginal como evento contextualizado culturalmente reflete os valores de cada sociedade humana, vindo dos sentidos atribuídos a ele a ao processo doloroso com fenômenos universais que transcendem a experiência física.

Portanto, a dor no parto normal é como uma experiência inerente ao processo de parturição e sua história está relacionada à ideia de sofrimento, e um aspecto cultural que está contribuindo para que o parto normal tenha conotação e significado de experiência traumática para a mulher, colaborando para a difusão do ideário de dor como sofrimento no imaginário feminino popular (ALMEIDA et al., 2012).

A dor no parto pode ser conceituada segundo a classificação geral de dor, como orgânica, aguda, transitória, complexa, subjetiva e multidimensional, que resulta em estímulos sensoriais gerados principalmente pela contração uterina. Podendo a dor ser associada a uma característica intermitente que é iniciada no trabalho de parto, e vai aumentando progressivamente segundo o padrão de contração uterina e é finalizada com o processo de nascimento (ALMEIDA et al., 2012).

Segundo a Organização Mundial de Saúde (OMS) o Brasil é o país que possui o maior índice de parto cesárea no mundo, com cerca de $43 \%$, sendo que o índice recomendado é somente $15 \%$. Este alto índice pode estar relacionado ao receio da dor do parto, a preocupação com a integridade da vagina e do períneo, podendo, também, ser a opção dos médicos pela praticidade e conveniência, devido a ser uma intervenção programada ao contrário do parto vaginal (LUIS et al., 2017).

É certo dizer que o fisioterapeuta possui um papel fundamental, pois ele atua preparando a gestante para o parto normal, orientando-a sobre a função dos músculos do assoalho pélvico, as posições que podem aliviar as dores e exercícios respiratórios. O fisioterapeuta estuda todos os movimentos do corpo humano, e com isso é um dos profissionais com mais capacidade para auxiliar positivamente no parto normal, onde a gestante precisa de mobilidade pélvica e uma intensa utilização da sua musculatura do abdômen, do períneo e do diafragma. Este tema foi escolhido no intuito de estudar a intervenção do fisioterapeuta no auxilio durante o parto normal, frisando a porcentagem de partos normais no Brasil que são menores comparados aos partos cesáreos (TAKEMOTO et al., 2013).

Com isso surge a seguinte problemática: Qual o papel do fisioterapeuta no auxílio do parto natural em gestantes?. Este artigo tem o objetivo de aborda à importância do fisioterapeuta no parto natural em 
gestantes, e como o mesmo pode preparar a mesma para o parto, observando ainda como o Brasil está em relação à porcentagem de partos normais.

\section{METODOLOGIA}

O presente artigo foi realizado através de um levantamento bibliográfico, realizada por meio de uma revisão de literatura de caráter qualitativo considerando a relevância do tema. A pesquisa foi realizada nos períodos de Fevereiro a Abril de 2018 pesquisando artigos na biblioteca virtual em Saúde: LILACS (Literatura Latino- americana e do Caribe em Ciências da Saúde), SciELO (Scientific Eletronic Library OnLine), Google Acadêmico e Arquivos de dissertações selecionando trabalhos em português para o desenvolvimento do tema abordado.

Para a pesquisa virtual, fez-se uso e combinação dos seguintes descritores: Parto natural; Gestação; Fisioterapia; Humanização. Dos artigos selecionados foram extraídas informações a dor no parto natural em gestantes, enfatizando o papel dos fisioterapeutas no auxílio e preparação de gestantes para o parto normal, relacionando a situação de porcentagem e percepção do parto natural no Brasil.

\section{REVISÃO TEÓRICA}

\section{Parto Natural}

O parto é um evento que já passou por muitas mudanças no decorrer dos anos. Antigamente, não existiam técnicas para diminuir a dor no parto e exercícios que favoreciam esta atividade. Por sua vez, as mulheres antigamente se isolavam para parir e geralmente sem nenhuma assistência ou cuidado, apenas seguiam seus extintos. A assistência ao parto teve início quando as mulheres começaram a se ajudar neste processo, com a participação de seus familiares e parteiras, que com isto foram acumulando experiências e passando de geração em geração. O parto foi institucionalizado após a segunda Guerra Mundial pela incorporação de novas experiências e habilidades aprendidas pelos médicos nos campos da assepsia, cirurgia, anestesia e hemoterapia (VIANA et al., 2014).

O conceito de parto normal está atribuído ao que ocorre naturalmente como um fenômeno natural. Para que isto aconteça e possa ser considerado um parto normal, o mesmo tem que ser realizado de modo que não ocorra intercorrências ou procedimentos desnecessários ao longo do trabalho de parto. $\mathrm{O}$ trabalho de parto é o momento em que a mulher está prestes a comtemplar o nascimento do seu bebê. Este é um período em que a mulher sente muita dor, na qual é causada pelas contrações que diminuem o fluxo sanguíneo uterino (VICENTE et al., 2017).

O trabalho de parto é dividido em duas fases, na qual a primeira é caracterizada por contrações uterinas que permitem a dilatação progressiva do colo uterino. Nessa fase ocorre muito estresse emocional como a dor. E a segunda fase é constituída pela a expulsão fetal, na qual as contrações e dilatações do colo uterino se tornam mais intensas. Com o avanço do trabalho de parto é caracterizado pela cervicodilatação uterina, que é realizado o toque vaginal. A progressão da dilatação cervical aumenta progressivamente a 
partir dos $4 \mathrm{~cm}$, que é a fase ativa do trabalho de parto, quando a velocidade da dilatação cervical está de $1 \mathrm{~cm}$ por hora (VICENTE et al., 2017).

\section{Percepções relacionadas à dor no parto normal}

A gravidez é um momento singular na vida das mulheres, ocasionando importantes mudanças físicas e emocionais, as quais podem ser intrinsecamente relacionadas aos contextos culturais, socioeconômicos e também ao modelo assistencial de saúde (ALMEIDA et. al, 2011). O incomodo no parto normal é associada a uma ideia de sofrimento, é um componente cultural que tem contribuído para que o parto natural tenha conotação e significado de experiência traumática para a mulher, e também tem ajudado para a difusão de ideário de dor como no psicológico feminino popular. Essa dor é bastante discutida atualmente no mundo com o propósito de subsidiar a assistência obstétrica e assim aumentar a satisfação da mulher na vivencia do processo parturitivo e favorecer a volta da prática do parto normal (ALMEIDA et al., 2012).

A Organização Mundial da Saúde e o Ministério da Saúde, preocupados com as questões do aumento das intervenções no processo natural do parto, programaram uma mudança de paradigma de assistência obstétrica de intervencionista para humanista. Esse humanismo no parto tem como objetivo a efetividade, qualidade e a segurança para os direitos de cidadania para a garantia da satisfação da parturiente no parto (GUEDES et. al., 2017).

As informações sobre o parto mesmo nos tempos atuais, ainda são bastante difundidas, o momento do parto é caracterizado por sentimentos de angustia, medo e fantasias pode parte das gestantes. Tornando assim a preparação para o trabalho de parto de grande relevância para que a parturiente sinta-se mais segura (LIMA et al., 2010).

\section{Vantagens e Desvantagens do Parto normal}

O parto normal possui diversos benefícios no qual oferece a mãe uma melhor recuperação, em um curto período de tempo, que também a mesma contará com menos riscos de sofrer infecções, hemorragias e lesões de órgãos, podendo também contribuir para que a gestante ingira menos medicamentos, diminuindo assim o risco de trombose. A perda de sangue no parto normal é menor, devido a não serem necessários cortes profundos e nem muitos grandes. No parto natural, a gestante perde somente meio litro de sangue; já na cesárea, essa quantia aumenta para 1,5L. Contudo, há vários benefícios para o bebê, como a criação de um vínculo ainda maior com a mãe. Mesmo passando por algum estresse durante esse tipo de parto, o recém-nascido desenvolve algumas substâncias em seu organismo que ajudam a prepará-lo para o ambiente diferente (MELO et al., 2015).

Outra vantagem do parto normal é que favorece a mãe na amamentação e os níveis de hormônios maternos elevam-se automaticamente com o trabalho de parto, no qual irá produzir colostro que é um liquido amarelo que antecede o leite, rico em anticorpos. Não há dor pós-parto, e a alta do hospital torna-se mais rápida, o que irá auxiliar a mãe a retornar o seus afazeres prontamente. A gestante irá participar 
ativamente do nascimento do seu filho, interagindo e construindo um grande vínculo com seu bebê (MELO et al., 2015).

Podemos citar também o benefício de não haver cicatriz aparente, devido a não haver a necessidade de uma incisão cirúrgica. Portanto, com o parto normal, diminuir-se-á possíveis implicações para futuros partos, como acontece em repetidas cesáreas. Para o bebê poderá, também, favorecer na saída dos líquidos pulmonares, o qual irá minimizar os riscos de problemas respiratórios. O nascimento por parto normal acontece no período certo, isto também diminui os riscos de retirada do feto quando o mesmo ainda não está preparado para nascer. Nesse tipo de parto, o feto começa a se adaptar com o ambiente exterior, acomodando o seu sistema fisiológico a um novo ritmo, de uma maneira suave, ou seja, em um ritmo natural (SILVA et al., 2014).

A parturiente, neste tipo de parto, poderá se movimentar em poucas horas, favorecendo a minimização de riscos de fenômenos tromboembólicos, melhorando o funcionamento intestinal e acelerando a recuperação física; aumenta os níveis de bem-estar da mãe, podendo ainda favorecer na produção de leite materno, o qual é de suma relevância para a constituição de um forte sistema imunitário no recém-nascido.

Essa produção de leite é ocasionada primeiramente pela contração do útero e com a submissão de uma cesariana este processo torna-se 'tardio' devido a saltar algumas etapas, e em um parto cesáreo quem irá estimular essa subida de leite será o bebê. Por fim, pode-se citar que nas primeiras 4 horas após o parto, os sinais vitais maternos se regularizam e os músculos uterinos inicia um processo de contração, ocasionado uma volta mais rápida do tamanho normal do útero, denominada involução uterina (SILVA et al., 2014).

De uma forma geral, o parto normal não possui quase nenhuma desvantagem. Quando não é possível realizá-lo devido a uma condição de saúde da gestante ou a do bebê não estar de cabeça para baixo, o médico irá informar à parturiente que será necessário à submissão de uma cesárea, ao invés do parto normal. Mas, mesmo não possuindo quase nenhuma desvantagem, o parto normal também poderá trazer alguns riscos, devido a isto é fundamental que a equipe médica oriente a gestante e informe caso seja preciso optar pela cesárea, para oferecer uma maior segurança a mãe e o bebê (CARAVLHO et. al., 2010).

Outra desvantagem para a mãe que podemos citar seria possíveis danos à pelve, ao períneo, uretra e ânus. Podendo causar uma incontinência urinária e fecal, causando também uma dor no períneo, em casos de episiotomia e laceração. A maioria dos danos ocorridos durante o parto normal é ocasionado pela má condução do mesmo, principalmente quando são utilizadas manobras e intervenções desnecessárias (MURAYAMA, 2014).

Uma desvantagem do parto normal citada por muitas mulheres, a qual tem ocasionado o aumento de parto cesáreos no Brasil, seria a dor causada pelas fortes contrações e o medo de complicações e desconforto quando é realizado a episiorrafia (que é a cicatriz resultante do corte no períneo, realizada com o intuito de aumentar a abertura vaginal durante o parto, para evitar as lacerações extensas), principalmente quando inflama no local (CARNEIRO et. al., 2015). 
O ideal seria que toda gestante, ao longo de sua gravidez, praticasse atividades físicas orientadas por um profissional qualificado, pois isto ajudaria bastante no trabalho de parto e em sua rápida recuperação no pós-parto. Entende-se que, quanto mais ativa a mulher se mantém, melhor será a evolução de seu parto, e assim conhecerá melhor seu corpo, e saberá realizar as posturas e padrões respiratórios mais indicados e confortáveis (GUEDES et al., 2017).

\section{Papel do fisioterapeuta no auxilio as gestantes no Parto normal}

Estudos evidenciam que desde a década de 70 a utilização de posturas verticais e da mobilidade materna durante o trabalho de parto é relevante e segura, para que ocorram movimentos de ajudem o encaixe do feto à pelve materna e ao canal de parto. Estes movimentos podem ajudar a minimizar a duração do trabalho de parto e aumentar a tolerância das dores. Os recursos mais eficazes são os realizados por fisioterapeutas que são: ajoelhada, quatro apoios, posição ortostática com inclinação de tronco, sentada, sempre respeitando a posição que a mulher sente-se mais confortável (SILVA et al., 2015).

A atuação do fisioterapeuta no acompanhamento ao trabalho de parto ainda não é incluída pelo sistema de saúde, mas é uma prática estabelecida na sociedade. Portanto, esse profissional tem uma relevante função de orientar e conscientizar a gestante para que a mesma desenvolva sua potencialidade que irá exigir no momento do parto, com mais confiança e segurança (SOUSA et al., 2014).

O auxílio do fisioterapeuta na assistência obstétrica de baixo risco irá valorizar a responsabilidade da gestante no processo, com a utilização ativa do próprio corpo. A mobilidade corporal no processo de parturição irá envolver diversos fatores fisiológicos, psicológicos, culturais e principalmente o apoio e a orientação da equipe obstétrica. $\mathrm{O}$ importante papel desse profissional é um aspecto importante para a gestante se conscientizar de que seu corpo ativo é uma excelente ferramenta para facilitar o processo do trabalho de parto e proporcionar a satisfação com a experiência do nascimento (BAVARESCO et al., 2011).

Outro relevante papel do fisioterapeuta é a promoção do conforto a parturiente, mostrando a ela as vantagens do parto natural, proporcionando segurança nestes procedimentos, que visam evitar cirurgias cesarianas. Compete a estes profissionais fazer um preparo individual das gestantes, para que possam ensina-las sobre a utilização da musculatura do assoalho pélvico, das melhores posições que minimizam as dores, e das técnicas respiratórias que podem ajudar no parto com um preparo psicológico da mulher, para que o mesmo ocorra da forma mais natural possível (BARROS et al., 2017).

O papel do fisioterapeuta no trabalho de parto e no parto é relevante à medida em que cada orientação e intervenção é empregada de acordo com a exigência do período funcional do trabalho de parto. As técnicas realizadas pelos fisioterapeutas incluem a postura, mobilidade, alternância de posições e respiração fisiológica, com o intuito de promover uma segurança e conforto para que a gestante sinta-se segura e acolhida. Também são utilizados recursos no objetivo da analgesia, como a massoterapia e as técnicas de relaxamento associadas ou não a exercícios de respiração (BARROS et al., 2017).

Podem-se citar as medidas não farmacológicas que são utilizadas pelos fisioterapeutas, como a massagem que é uma excelente forma para promover o alivio da dor, ocasionando o relaxamento muscular, 
tendo o efeito sedativo e analgésico. Há uma resposta importante através da massagem que é a liberação de endorfina. As endorfinas podem diminuir as transferências de sinais entre as células nervosas, diminuindo e causando uma tolerância maior a dor (RODRIGUES, 2007). Essa estimulação provocada pela massagem é detectada pelo cérebro, podendo percorrer outras partes do corpo, promovendo uma melhoria na circulação, causando um aumento do suprimento de nutrientes para a placenta, devido ao favorecimento do transporte destas substâncias (ABREU et al., 2013).

Essas massagens são realizadas geralmente na região lombo- sacra e nas pernas, locais que as gestantes mais referem quadro álgico. A massagem feita na região perineal é muito eficaz, para as mulheres que expressam maiores riscos de lacerações. O relaxamento das fibras, a tensão local é aliviada com estas massagens realizadas pelos fisioterapeutas, no intuito de prevenir maiores lacerações na hora do parto e evitando a episiotomia (ANDRADE, 2016).

O fisioterapeuta deve estar atento no trabalho de parto e no parto para a percepção respiratória das gestantes. As técnicas mais utilizadas para a respiração são a torácica e a abdominal, na qual a respiração torácica é a mais indicada para o momento das contrações, devido à mesma ocasionar uma expansão torácica no sentido lateral, aliviando o fundo uterino e uma maior oxigenação. Já a respiração abdominal deve ser realizada nos intervalos das contrações, promovendo um maior relaxamento para a gestante (POGLITSCH et al., 2014).

Usando técnicas respiratórias promovidas pelos fisioterapeutas quando realizadas de forma correta, podem resultar em tranquilidade e relaxamento das parturientes. Algumas emoções também podem interferir na respiração, nas quais o fisioterapeuta deve instruir a parturiente a utilizar sua musculatura respiratória corretamente para ocasionar uma melhor oxigenação para o bebê e para si própria (MARTINS et al., 2013).

Outra técnica muito empregada pelo fisioterapeuta é a utilização da bola suíça, que tem o intuito de promover uma livre movimentação da mulher durante o parto. A utilização dessa bola pode ocasionar uma correção na postura, relaxamento, alongamento e o fortalecimento da musculatura. Quando são realizados exercícios com a bola suíça na posição sentada, pode fazer com que haja uma movimentação suave da pelve, ocasionando o relaxamento e contribuindo para o processo de nascimento do bebê. A bola suíça é uma técnica utilizada para distrair a gestante, possibilitando um parto mais tranquilo (VALENCIANO et al., 2015).

\section{RESULTADOS E DISCUSSÃO}

Notou-se uma grande rejeição por parte das mulheres em optarem pelo parto normal, devido às dores sentidas no trabalho de parto e no parto. De acordo com Velho et al. (2012), o parto normal é associado pelas mulheres como um processo doloroso, com intensidade de dor maior do que a esperada, mesmo que por pouco tempo. Muitas parturientes sentem medo quando pensam no parto normal, de que alguma complicação possa acontecer com o bebê ou até consigo mesmo.

Portanto, é nítida a grande quantidade de mulheres que estão optando por partos cesáreos, pois segundo Silva et al. (2014), as mesmas sentem receio de parir por parto normal por temerem as 
consequências que possam ter, como o desencadeamento de incontinência urinária e fecal e até lacerações perineais. Este medo pode ser ainda maior quando não há um diálogo com profissionais de saúde que possam acompanha-las no pré-natal, esclarecendo dúvidas em relação à escolha do tipo de parto, esta aproximação entre a gestante e o profissional tem o objetivo de fornecer informações que minimizem a ansiedade e insegurança das mulheres.

Porém, perceberam-se também pontos relevantes na optação pelo parto normal. De acordo com Scarton et al. (2018), o parto normal traz benefícios para mãe a para o bebê, pois o mesmo minimiza os riscos para a saúde de ambos, em gestações de baixo risco, além de o parto natural ser recomendado pela Organização Mundial de Saúde (OMS). O parto normal pode auxiliar para uma recuperação mais rápida e sem dores após o parto, permitindo ainda na interação plena com o bebê, desde o primeiro minuto do seu nascimento, criando um vínculo.

Scarton et al. (2018) afirma ainda que o parto normal traz grandes benefícios para o bebê, no qual poderá favorecer a maturidade pulmonar e irá prevenir complicações respiratórias. O bebê irá nascer mais ativo e terá maiores chances de se alimentar exclusivamente do leite materno sobre livre demanda. Relatando ainda que ao passar pelo canal vaginal, a flora bacteriana da mãe passa para o bebê, o auxiliando a formar sua própria flora intestinal.

Segundo dados divulgados pelo Ministério da Saúde em 2016, dos 3 milhões de partos feitos no Brasil neste período, cerca de 55,5\% foram cesáreas e $44,5 \%$ foram partos normais. Ainda de acordo com o ministério da saúde algo que chamou bastante a atenção é que de 2013 a 2016, o número de partos normais subiu de $43 \%$ para $44,5 \%$ enquanto o de cesáreas caiu de $57 \%$ para $55 \%$. Todavia, o número de cesarianas no país ainda é significativo (VICENTE et al., 2017).

Com isso, é visto que há uma grande necessidade da participação ativa do fisioterapeuta no auxílio ao parto normal, para que haja mais um conforto por parte das gestantes e parturientes. De acordo com Duarte et al. (2012), para o alívio da dor no parto para as parturientes, os cuidados não farmacológicos são necessários para substituir a utilização de anestésicos e analgésicos durante o trabalho de parto, sendo que a dor que a parturiente sente durante o trabalho de parto e no parto é influenciado por vários fatores como o medo e a ansiedade e é único para cada mulher. O trabalho do fisioterapeuta está em relacionar os conceitos da fisiologia e da biomecânica com a escola de processos corretos para cada parturiente. Portanto, o intuito da fisioterapia é minimizar o período de dilatação, tornando o mesmo mais tranquilo, e ainda preparando o períneo para o período expulsivo.

Silva et al. (2012) relata ainda que o fisioterapeuta durante o atendimento a gestante deve organizar exercícios de relaxamento para a facilitação da dilatação, o qual irá acarretar uma melhor evolução e uma qualidade de vida proporcionada à parturiente e ao bebê. O fisioterapeuta ainda pode orientar a parturiente sobre as diversas formas de diminuir as tensões musculares com posturas que auxiliem na dilação, prosseguindo com informações sobre a postura e exercícios de respiração adequada para ajudá-la na expulsão do feto. Há uma grande relevância na atuação do fisioterapeuta em aspectos distintos, como o lado psicológico, humano e social, no qual a principal área de atuação deste profissional é a mobilização corporal 
realizada com movimentos amplos e específicos, com o intuito de levar a parturiente a um trabalho de parto satisfatório.

$\mathrm{Na}$ qual se pode ainda salientar que a fisioterapia obstétrica como é configurada atualmente tem o objetivo de proporcionar a gestante um maior conforto e bem-estar na gestação e no pós-parto, promovendo condições que acelere as contrações uterinas levando a um trabalho de parto mais rápido e menos doloroso. Observando a necessidade de palestras e dinâmicas de grupos que visam solucionar ou desmistificar certos conceitos errôneos que ainda possuem (LACERDA et al., 2016).

Apesar da limitação do presente estudo ter como critério estudos apenas na língua portuguesa, mostrou que o fisioterapeuta tem um importante papel no trabalho de parto e no parto, trazendo benefícios para as pacientes, acredita-se que este estudo pode trazer benefícios para o esclarecimento de dúvidas com relação às dores no parto normal. Percebe-se ainda a necessidade de frisar o trabalho dos fisioterapeutas no atendimento a gestantes e parturientes, sendo necessários novos estudos sobre o tema, para que assim seja abordado ainda mais a relevância da atuação deste profissional nesses casos, ressaltando ainda informações sobre o parto normal, promovendo uma influência para que as gestantes optem pelo parto natural, o qual traz mais benefícios para a mãe e o bebê.

\section{CONSIDERAÇÕES FINAIS}

Notou-se, com esta pesquisa, que a fisioterapia pode auxiliar para a qualidade do trabalho de parto, contribuindo para facilitar em todas as suas fases, como técnicas que podem ajudar para o alivio da dor, permitindo que a parturiente tenha uma melhor consciência corporal, prevenindo ou diminuindo lesões no assoalho pélvico, e auxiliando na recuperação no pós-parto. É papel do fisioterapeuta dar o esclarecimento às gestantes quanto aos tipos de parto e todas as possibilidades de parto existentes. Portanto, é de suma relevância que haja esclarecimentos para as gestantes, afim de que as mulheres estejam preparadas e devidamente acompanhadas no intuito de contribuir positivamente para a evolução do parto, minimizando o tempo de internação e infecções hospitalares.

Percebeu-se também que é função do fisioterapeuta ser especializado em saúde da mulher para o trabalho com gestantes, auxiliando e orientando para que as mesmas estimulem uma potencialidade do seu corpo no trabalho de parto e no parto, para que não ocorra nenhum tipo de complicações, podendo levar a contrações erradas ou pouco efetivas. A abordagem fisioterapêutica terá resultados benéficos sobre a qualidade de vida da gestante, ajudando e prevenindo maiores lesões na musculatura do assoalho pélvico nos partos normais, dando informações sobre as posturas corretas a serem tomadas durante a gravidez e pós-parto.

Observou-se que o acompanhamento fisioterapêutico durante a gestação reduz a prevalência de algias, controle da ansiedade, auxilia no entendimento das fases da gestação, minimiza o tempo de internação, no intuito de diminuir os gastos públicos com internações prolongadas em função de lesões perineais e intervenções cirúrgicas, trazendo vários benefícios para os músculos. 
É relevante que haja uma maior promoção de estudos que informe as mulheres sobre as dores no parto normal, para que seja percebida de forma significativa pelos profissionais. Como já citado é de grande importância à atuação do fisioterapeuta nas maternidades, auxiliando nas salas de parto normal, favorecendo um atendimento obstétrico acolhedor, garantindo a satisfação da mãe e do bebê. Há uma grande necessidade de realizar mais estudos que possam possibilitar uma melhor percepção das gestantes e dos gestores de saúde, para a inserção do fisioterapeuta na equipe multidisciplinar, na qual irá somente acrescentar na unidade hospitalar e ajudar as parturientes que precisam de auxílio para um parto satisfatório no ponto de vista fisioterapêutico.

O fisioterapeuta é o profissional da saúde com maior capacidade e conhecimento para oferecer uma estrutura de forma segura e eficiente, com métodos não farmacológicos para o alivio da dor no trabalho de parto e durante o parto. Portanto há uma grande influência deste profissional para acrescentar confiança e segurança para as parturientes no intuito de ocorrer uma evolução no parto, acarretando maiores benefícios para a redução da duração do trabalho de parto.

Por fim, é certo afirmar que o parto normal possui bastantes vantagens, de extrema relevância para o bem- estar da mãe e do bebê. Com relação a grande quantidade relatada em estudos de gestantes que optam por parto Cesário, há uma grande necessidade de intensificar a divulgação com uma conscientização sobre as vantagens do parto normal que por sua vez é mais saudável.

\section{REFERÊNCIAS}

ALMEIDA, M. R. C. B.; SOUZA, C. V.; MACIEL, V. B.; RIBEIRO, J. T.; AMARO, M. L. M.; COSTA, M. J. P.; STRAPASSON, S.. Percepção e perspectivas de gestantes sobre o processo do parto a partir de oficinas educativas. Revista Mineira de Enfermagem, Belo Horizonte, v.15, v.1, p.79-85, 2011.

ABREU, N. S.; CRUZ, M. V.; GUERRA, Z. F.; PORTO, F. R. Atenção Fisioterapêutica no Trabalho de Parto e Parto. Revista Interdisciplinar de Estudos Experimentais, Juiz de Fora, v.5, p.7-15, 2013.

ALMEIDA, N. A. M.; MEDEIROS, M.; SOUZA, M. R. Perspectivas de dor do parto normal de primigestas no período pré-natal. Revista Texto \& Contexto Enfermagem, Florianópolis, v.21, n.4, p.819-827, 2012.

ANDRADE, M.. Parto Normal e Fisioterapia. São Paulo: 2016.

BAVARESCO, G. Z.; SOUZA, R. S. O.; ALMEICA, B.; SABATINO, J. H.; DIAS, M.. O fisioterapeuta como profissional de suporte à parturiente. Ciência \& Saúde Coletiva, Florianópolis, v.16, n.7, p.3259- 3266, 2011.

BARROS, A. P.; MATOS, S. S.. A Importância da Atuação do Fisioterapeuta no Parto Vaginal em Primigestas e Multíparas. Revista Científica Multidisciplinar Núcleo do Conhecimento, Porto Velho, v.1, n.6, p.282-291, 2017.

CARNEIRO, L. M. A.; PAIXÃO, G. P. N.; SENA, C. D.; SOUSA, A. R.; SILVA, R. S.; PEREIRA, A.. Parto Natural X Parto Cirúrgico: percepções de mulheres que vivenciaram os dois momentos. Revista de Enfermagem do Centro Oeste Mineiro, São João Del-Rei, v.5, n.2, p.1574-1585, 2015;
CARVALHO, V. F.; KERBER, N. P. C.; BUSANELLO, J.; COSTA M. M. G.; GONÇALVES, B. G.; QUADROS, V. F.. Práticas prejudiciais ao parto: relato de trabalhadores de saúde do sul do Brasil. Revista da Rede de Enfermagem do Nordeste, Fortaleza, v.11, p.92-98, 2010.

GIL, A. C.. Como elaborar projeto de pesquisa. 4 ed. São Paulo: Atlas, 2002.

GUEDES, C. D. F. S.; SOUZA, T. K. C.; MEDEIROS, L. N. B.; SILVA, D. R.; NETA, B. P. A. A.; SANTOS, M. M.; COSTA, A. B.. Percepções de gestantes sobre a promoção do parto normal no pré-natal. Revista Ciência Plural, Natal, v.3, n.2, p.87-98, 2017.

LUIS, L. A.; SANTOS, P. C. O.; MATCUMOTO, T. A. S.; AQUINO, S. R. J.. Intervenção Fisioterapêutica no Preparo e Durante o Parto. Monografia (Graduação em Fisioterapia) Faculdades Integradas de Fernandópolis, Fernandópolis, 2017.

LACERDA, A. F.; SILVA, A. C.; URQUIZA, C. N.; PEREIRA JÚNIOR, J. L.; CHIARAPA, T. R. Assistência fisioterapêutica na sala de parto. Monografia (Graduação em Fisioterapia) Universidade Católica Dom Bosco, Campo Grande, 2016.

LIMA, L. A.; BERETTA, M. S.. O papel do fisioterapeuta no programa de humanização no pré-natal e nascimento: uma proposta para prefeitura de Bragança Paulista. Monografia (Graduação em Fisioterapia) - Universidade São Francisco, Bragança Paulista, 2010. 
MELO, J. K. F.; DAVIM, R. M. B.; SILVA, R. R. A.. Vantagens e Desvantagens do parto normal e cesariano: opinião de puérperas. Revista de Pesquisa Cuidado é Fundamental, Rio de Janeiro, v.7, n.4, p.3197-3205, 2015.

MURAYAMA, B.. Parto Natural: vantagens e desvantagens. São Paulo: 2014.

MARTINS, A. A.; LEAL, L. G.. A importância da fisioterapia no parto natural. Goiânia: 2013.

POGLITSCH, F. A. P. B.; SHUMAN, S.. Parto humanizado: intervenção da fisioterapia na equipe multidisciplinar. São Paulo: 2014.

RODRIGUES, E.. Intervenção fisioterapêutica na assistência ao trabalho de parto. Dissertação (Mestrado em Ciências) Universidade de São Paulo, São Paulo, 2007.

SILVA, T. F.; MEJIA, D. P. M.. Relevância da fisioterapia no período gestacional. Goiânia: 2012.

SILVA, C. F.; LUZES, R.. Contribuição da Fisioterapia no Parto Humanizado: revisão de literatura. Revista Discente da UNIABEU, v.3, n.6, p.25-32, Rio de Janeiro, 2015.

SCARTON, J.; RESSEL, L. B.; SIQUEIRA, H. C. H.; RANGEL, R. F.; TOLFO, F.; WEYKAMP, J. M.. Práticas de atenção ao parto normal: a experiência de primíparas. Revista Online de Pesquisa, Santa Maria, v.10, n.1, p.17-24, 2018.

SILVA, S. P. C.; PRATES, R. C. G.; CAMPELO, B. Q. A.. Parto normal ou cesariana? Fatores que influenciam na escolha da gestante. Revista de Enfermagem UFSM, Santa Maria, v.4, n.1, p.1-9, 2014.

SOUSA, D. P. F.; CUNHA, F. M. A. M.. A fisioterapia na preparação para o trabalho de parto: uma revisão integrativa. Revista Digital EFDeportes.com, Buenos Aires, v.19, n.195, 2014.

TAKEMOTO, A. Y.; CORSO, M. R.. Parto humanizado e a assistência de enfermagem: uma revisão da literatura. Revista Arquivos de Ciência Saúde, São José do Rio Preto, v.17, n.2, p.117-127, 2013.

VALENCIANO, C. M. V. S.; RODRIGUES, M. F.. a importância da intervenção fisioterapêutica na assistência do trabalho de parto. Monografia (Graduação em Fisioterapia) - Centro Universitário Católico Salesiano Auxilium, Lins, 2015

VELHO, M. B.; SANTOS, E. K. A.; BRÜGGEMANN, O. M.; CAMARGO, B. V.. Vivência do parto normal ou cesáreo: revisão integrativa sobre a percepção de mulheres. Revista Texto \& Contexto de Enfermagem, v.21, n.2, p.458-466, 2012.

VIANA, L. V. M.; FERREIRA, K. M.; MESQUITA, M. A. S. B.. Humanização do parto normal: uma revisão de literatura. Revista Saúde em foco, Teresina, v.1, n.2, p.134-148, 2014.

VICENTE, A. C.; LIMA, A. K. B.; LIMA, C. B.. Parto Cesário e Parto Normal: uma abordagem acerca de riscos e benefícios. Revista Temas em Saúde, João Pessoa, v.17, n.4, p.24-35, 2017.

A CBPC - Companhia Brasileira de Produção Científica (CNPJ: 11.221.422/0001-03) detém os direitos materiais desta publicação. Os direitos referem-se à publicação do trabalho em qualquer parte do mundo, incluindo os direitos às renovações, expansões e disseminações da contribuição, bem como outros direitos subsidiários. Todos os trabalhos publicados eletronicamente poderão posteriormente ser publicados em coletâneas impressas sob coordenação da Sustenere Publishing, da Companhia Brasileira de Produção Científica e seus parceiros autorizados. Os (as) autores (as) preservam os direitos autorais, mas não têm permissão para a publicação da contribuição em outro meio, impresso ou digital, em português ou em tradução. 